\title{
Estudo multicêntrico dos resultados das trocas valvares com o uso da bioprótese Biocor no Estado de Minas Gerais
}

\author{
Mário Osvaldo VRANDECIC*; Bayard GONTIJO FILHO*; João Alfredo Paula e SILVA*; Fernando Antônio \\ FANTINI*; Juscelino Teixeira BARBOSA ${ }^{\star \star}$; Márcio C. SÃO JOSÉ; Carlos Álvaro dos Santos PINTO***; \\ Gilberto Lino VIEIRA ${ }^{\star \star \star *}$; Homero Geraldo OLIVEIRA ${ }^{\star \star \star \star *}$; Renato R. RABELO ${ }^{\star \star \star \star * ; ~ S e b a s t i a ̃ o ~ C o r r e a ~ R A B E L-~}$ \\ LO $^{\star * \star *}$; Alexandre V. BRICK ${ }^{\star \star * \star *}$; Eduardo PEREDO*; Adelson A. PEDROSA*; Antônio Luiz O. AZEVEDO \\ SOBRINHO ${ }^{\star * *}$; Maurício BARBOSA ${ }^{\star \star \star}$; Heberth César MIOTTO*; Maria Aparecida BRAGA ${ }^{* \star * *}$; Marco Antô- \\ nio SALUM**; Júnia F. BRAGA*; Guilherme H. MOREIRA*; Osvald Hely MOREIRA*; Carlos Alberto \\ de OLIVEIRA***; Flávio Justo MACIEL*
}

\section{RBCCV 44205-62}

VRANDECIC, M. O.; GONTIJO FILHO, B.; SILVA, J. A. P.; FANTINI, F. A.; BARBOSA, J. T.; SĀO JOSÉ, M. C.; PINTO, C. A. S.; VIEIRA, G. L.; OLIVEIRA, H. G.; RABELO, R. R.; RABELLO, S. C.; BRICK, A. V.; PEREDO, E.; PEDROSA, A. A.; AZEVEDO SOBRINHO, A. L. O.; BARBOSA, M.; MIOTTO, H. C.; BRAGA, M. A.; SALUM, M. A.; BRAGA, J. F.; MOREIRA, G. H.; MOREIRA, O. H.; OLIVEIRA, C. A.; MACIEL, F. J. - Estudo multicêntrico dos resultados das trocas valvares com o uso da bioprótese Biocor no Estado de Minas Gerais. Rev. Bras. Cir. Cardiovasc., 3(3): 159-168, 1988.

RESUMO: No período de março de 1981 a março de 1988, foram implantadas 2324 biopróteses, em 2016 pacientes, em 5 Centros do Estado de Minas Gerais. Este estudo inclui somente a análise dos pacientes submetidos a troca valvar aórtica $(n=603)$ e mitral $(n=1110)$, isoladamente. Neste grupo $(n=1713)$, a mortalidade hospitalar foi de 104 pacientes $(6,1 \%)$. Dos 1609 pacientes que receberam alta do hospital, conseguimos o seguimento de 1101 pacientes $(64,3)$. Esta análise corresponde a um período de 1 a 84 meses, com média de 48 meses e com um seguimento cumulativo de: aórticas (n $=385)=1230$ pacientes/ano; mitrais $(n=716)=3018$ pacientes/ano. Foram registradas 102 complicações tardias em 716 pacientes mitrais $(14,24 \%)$ e 51 complicaçōes no grupo aórtico $(13,2 \%)$. Com relação à faixa etária, encontramos 220 pacientes menores de 20 anos (mitrais $=176$ /aórticos $=44$ ) e, neste subgrupo, as disfunçōes valvares incidiram em $43 \%$ dos pacientes mitrais e em $29 \%$ dos pacientes aórticos. A endocardite protética foi mais encontrada nos aórticos $(45 \%)$ do que nos mitrais $(29,7 \%)$. Dos 1101 pacientes, 62 foram reoperados, com mortalidade hospitalar de $12,6 \%$. A reoperação por disfunção valvar foi mais freqüente no grupo mitral com idade inferior a 20 anos. A curva atuarial livre de mortalidade relacionada à bioprótese foi de $97,1 \%$ (32/1101 pacientes). No grupo aórtico, 96,9\% estiveram livres de disfunção valvar ao final de 7 anos, enquanto que, no grupo mitral, este índice foi de $95,2 \%$. Nos pacientes menores de 20 anos do grupo mitral, encontramos o maior índice de falência valvar (85,3\% livre após 7 anos). Ao final deste estudo, a maior parte destes pacientes encontrava-se em classe funcional I e II. Embora a incidência de reoperação seja expressiva, esta é aceitável, tendo em vista que as biopróteses oferecem uma alternativa mais segura do que as próteses mecânicas, durante este mesmo período do seguimento. A análise destes resultados sugere a continuação de pesquisas que tornem possivel a fabricação de um substituto valvar ideal. Todavia, estes resultados comparam-se, de modo favorável, a resultados com biopróteses semelhantes da literatura mundial.

DESCRITORES: próteses valvulares cardíacas, biológicas; próteses valvulares cardíacas, cirurgia; valvas cardiacas, cirurgia.

\footnotetext{
Trabalho realizado na Santa Casa de Misericórdia de Belo Horizonte, no Hospital Vera Cruz, Prontocor, Biocor Hospital de Doenças Cardiovasculares de Belo Horizonte e na Santa Casa de Juiz de Fora, MG, Brasil.

Apresentado ao 15: Congresso Nacional de Cirurgia Cardiaca. Rio de Janeiro, RJ, 8 e 9 de abril, 1988.

- Do Biocor Hospital de Doenças Cardiovasculäes.

-. Da Santa Casa de Misericórdia de Belo Horizonte.

... Do Prontocor.

.... Do Hospital Vera Cruz.

.... Da Santa Casa de Juiz de Fora.

Endereço para separatas: Mário Vrandecic. Rua Prof. António Aleixo, nং 307. 30180 Belo Horizonte, MG, Brasil.
} 
VRANDECIC, M. O.; GONTIJO FILHO, B.; SILVA. J. A. P.; FANTINI, F. A.; BARBOSA, J. T.: SÃO JOSÉ, M. C.; PINTO, C. A. S.; VIEIRA, G. L.; OLIVEIRA, H. G.: RABELO, R. R.; RABELLO, S. C.; BRICK, A. V.; PEREDO, E.; PEDROSA, A. A.; AZEVEDO SOBRINHO, A. L. O.; BARBOSA, M.; MIOTTO, H. C.; BRAGA, M. A.; SALUM, M. A.; BRAGA, J. F.; MOREIRA, G. H.; MOREIRA, O. H.; OLIVEIRA, C. A.; MACIEL, F. J. - Estudo multicêntrico dos resultados das trocas valvares com o uso da bioprótese Biocor no Estado de Minas Gerais. Rev. Bras. Cir. Cardiovasc., 3(3): 159-168, 1988.

\section{INTRODUÇÃO}

O funcionamento das próteses em geral é afetado por vários fatores, destacando-se as características da população. A nossa realidade sócio-econômica, além de determinar um indice expressivo de doenças valvares em jovens, dada a alta prevalência de doença reumática, também dificulta o uso adequado de anticoagulantes, na maioria dos pacientes $1,2,3,26,42$. A escolha do substituto valvar fica restrita ao uso da prótese mecânica, se aceitarmos a alta incidência de complicações tromboembólicas, cuja incidência cumulativa, nos países desenvolvidos, varia de 4,6 a 6,4 por paciente/ano ${ }^{11}$. 12-16, 21, 22, 30, 34, 36, 45 , ou às biopróteses que apresentam uma baixa incidência de complicaçōes tromboembólicas, mesmo sem o uso de anticoagulantes, reconhecendo a sua limitação em termos de durabilidade $6,7,9,18,19$. $20,24,25,27,28,31,38$

As biopróteses cardíacas, em uso clínico há mais de 15 anos, motivaram a pesquisa e a fabricação nacional de uma prótese de suíno de baixo perfil, montada em anel de Celcon ${ }^{1}$ (acetal copolímero) previamente revestido de pano tubular de Dacron**, ao qual são inseridas 3 cúspides previamente curtidas com soluçāo de glutaraldeído***, desprovidas de banda muscular e obtidas a partir da seleçāo precisa de válvulas aórticas suínas ${ }^{\star \star \star *}$.

O objeto deste trabalho é a apresentação da experiência clínica com a bioprótese Biocor, em Minas Gerais, em 5 Centros que participaram deste estudo multicêntrico.

\section{MATERIAL E MÉTODOS}

De março de 1981 a março de 1988 , foram implantadas 2273 biopróteses em 2016 pacientes. A distribuição anatômica dos implantes encontrada foi: troca mitral em $1110(55,10 \%)$ pacientes; troca aórtica em 603 $(29,90 \%)$; troca mitro-aórtica em $249(12,39 \%)$; troca mitro-aórtica-tricúspide em $5(0,24 \%)$; troca mitro-tricúspide em $20(0,96 \%)$; troca tricuspídea em $19(0,95 \%)$ e troca pulmonar em $10(0,46 \%)$, totalizando 2016 pacientes $(100 \%)$. O diâmetro das biopróteses usadas demonstrou maior incidência da bioprótese de $25 \mathrm{~mm}$ em posição aórtica e de 29 e $31 \mathrm{~mm}$ em posiçăo mitral. Foram realizadas 1110 trocas mitrais e 603 trocas aórticas, totalizando 1713 pacientes, dos quais foram encontrados 1101 $(64,30 \%)$, o que representa o universo pesquisado. Os dados foram obtidos por informação do clínico, parente, hospital, ou clínica local, análise dos prontuários, ou exa-

\footnotetext{
- Celanese Plastic Materials Company, Bishop. Texas, USA

* U.S.C.I. Billerica. Massachusetts, USA.

*.. Polysciences, Inc. Pennsylvania, USA.

.... Biocor Indústria e Pesquisas Ltda. (1981)
}

me clínico recente por um dos membros participantes deste estudo multicêntrico. A distribuição etária variou de 4 a 82 anos (média de 34 anos), sendo que 220 pacientes $(20 \%)$ se encontravam na faixa etária até 20 anos. Não houve predominância significativa do sexo ( ठ 50,6 versus $949,4 \%$ ). A etiologia predominante na indicação cirúrgica foi seqüela de doença reumática $(67 \%)$. A classe funcional pré-operatória mostrou grande maioria dos pacientes em grupo funcional III e IV (77\%) e apenas $23 \%$ em classe funcional II.

\section{Procedimento Cirúrgico}

A técnica cirúrgica empregada pelos diversos grupos foi homogênea e constituiu na incisão ântero-lateral direita ou esternotomia mediana como acesso cirúrgico, uso de oxigenador de bolhas nacional e cardioplegia cristalóide. A utilização de pontos separados predominou, sendo raro o uso de sutura contínua. $O$ tempo de pinçamento aórtico foi de 15 a 75 minutos, com média de 25 minutos para troca única e de 50 minutos para as trocas duplas. $\mathrm{O}$ tempo médio de extracorpórea foi de 60 minutos.

\section{Uso de anticoagulantes}

O uso de anticoagulantes foi restrito apenas a pacientes com história prévia de embolia ou presença de trombos em átrio esquerdo. A fibrilação atrial não indicou o uso de anticoagulantes. Foi verificada total irregularidade no uso do mesmo, quando prescrito.

\section{Definição de Falência Valvular}

Os critérios usados para definir falência valvular foram: 1) aparecimento de novo sopro, excluindo a possibilidade de insuficiência paravalvular; 2) episódios embólicos e/ou tromboembólicos, determinando reoperaçāo ou morte; 3 ) endocardite (até 3 meses de alta hospitalar); 4) hemorragias secundárias ao uso de anticoagulantes; 5) disfunção valvular evidenciada pelo ecocardiograma ou estudo hemodinâmico, resultando em morte; 6 ) resultado de análise anatômica patológica do espécimen, quando possivel, usado para melhor definir a natureza da falência valvular; 7) tromboembolismo central ou periférico sem causa aparente.

\section{Seguimento e análise dos dados}

O levantamento dos dados foi concluído em 28 de fevereiro de 1988. Este estudo inclui somente a análise dos pacientes submetidos a troca valvar aórtica $(n=$ 603 ) e mitral ( $n=1110$ ), isoladamente. Neste grupo 
VRANDECIC, M. O.; GONTIJO FILHO, B.; SILVA, J. A. P.; FANTINI, F. A.; BARBOSA, J. T.; SĀO JOSÉ, M. C.; PINTO, C. A. S.; VIEIRA, G. L.; OLIVEIRA, H. G.; RABELO, R. R.; RABELLO, S. C.; BRICK, A. V.; PEREDO, E.; PEDROSA, A. A.; AZEVEDO SOBRINHO, A. L. O.; BARBOSA, M.; MIOTTO, H. C.; BRAGA, M. A.; SALUM, M. A.; BRAGA, J. F.; MOREIRA, G. H.; MOREIRA, O. H.; OLIVEIRA, C. A.; MACIEL, F. J. - Estudo multicêntrico dos resultados das trocas valvares com o uso da bioprótese Biocor no Estado de Minas Gerais. Rev. Bras. Cir. Cardiovasc., 3(3): 159-168, 1988.

( $n=1713$ ) a mortalidade hospitalar foi de 104 pacientes $(6,1 \%)$. Dos 1609 pacientes que receberam alta hospitalar, conseguimos o seguimento de 1101 pacientes $(64,3 \%)$. Com relação à faixa etária, encontramos 220 pacientes menores de 20 anos (mitrais $=170$ /aórticos $=40$ ).

\section{Método Estatístico}

As estimativas atuariais foram analisadas usando o método do produto linear de Kaplan-Meir, assim como o teste de Gehan na avaliaçāo da incidência das complicaçōes relacionadas ao tempo. A análise deste grupo inclui seguimento de 1 a 84 meses, média 48 meses, cumulativo de 3018 para os pacientes mitrais e de 1230 para os aórticos.

TABELA 1

COMPLICAÇŌES DETERMINANTES DO ÓBITO HOSPITALAR

\begin{tabular}{|c|c|c|c|c|c|}
\hline OPERATÓRIAS & $\begin{array}{l}\text { N. DE } \\
\text { CASOS }\end{array}$ & C.T.I. & $\begin{array}{l}\text { N:DE } \\
\text { CASOS }\end{array}$ & ENFERMARIA & $\begin{array}{l}\text { N:DE } \\
\text { CASOS }\end{array}$ \\
\hline \multirow{7}{*}{$\begin{array}{l}\text { Embolia central } \\
\text { Falência biventricular } \\
\text { Perfuração ventricular } \\
\text { posterior } \\
\text { Discrasia sangüínea } \\
\text { fulminante }\end{array}$} & 1 & A.V.C. & 5 & Infecção pulmonar & 6 \\
\hline & 7 & Discrasia sangüinea & 4 & Embolia pulmonar & 8 \\
\hline & & & & i & \\
\hline & 2 & Falência biventricular & 24 & Insuficiência cardiaca & 7 \\
\hline & & & & & \\
\hline & 6 & Infecção pulmonar & 19 & Arritmias & 8 \\
\hline & & Insuficiência renal & 6 & Mediastinite & 1 \\
\hline TOTAL $\quad 104$ & 16 & & 58 & . & 30 \\
\hline
\end{tabular}

A mortalidade hospitalar geral foi de $6,1 \%$. As complicaçōes determinantes do óbito hospitalar (Tabela 1)

\begin{tabular}{|c|c|c|c|c|c|}
\hline 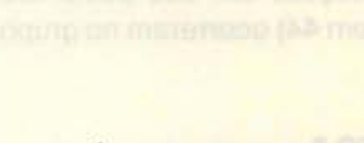 & $102 \mathrm{COM}$ & $\begin{array}{r}\text { TA } \\
\text { GRUI } \\
A C O E S T \\
D / S T\end{array}$ & $\begin{array}{l}\text { A } 2 \\
\text { IITRAL } \\
\text { IAS EM } 716 \text { PACIENTES } \\
\text { IÇAOO }\end{array}$ & 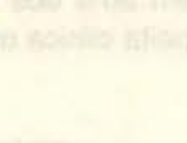 & \\
\hline$A T E ́<20$ ANOS IDADE & $N: D E$ & PERC. & $A P O S>20$ ANOS IDADE & $N: D E$ & PERC. \\
\hline 176 PACIENTES & PACIENTES & $\%$ & 485 PACIENTES & PACIENTES & $\%$ \\
\hline Endocardite & 18 & 29,7 & Endocardite & 10 & 25 \\
\hline Calcificação & 26 & 43 & Calcificação & 8 & 20 \\
\hline Embolia & 3 & 6 & Embolia & 6 & 14 \\
\hline Insuf. paravalvular & 4 & 6,3 & Insuf. paravalvular & 6 & 16 \\
\hline Insuf. cardiaca & 4 & 6,4 & Insuf. cardíaca & 10 & 20 \\
\hline Arritmias & 4 & 6,3 & Arritmias & 2 & 5 \\
\hline TOTAL & 60 & 100 & TOTAL & 42 & 100 \\
\hline
\end{tabular}

\begin{tabular}{|c|c|c|c|c|c|}
\hline 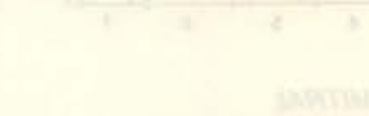 & 51 COMP & $\begin{array}{r}\text { GRUP } \\
C O E S T \\
\text { DIST }\end{array}$ & $\begin{array}{l}\text { SEM } 385 \text { PACIENTES } \\
\text { CAOO }\end{array}$ & & \\
\hline $\begin{array}{c}\text { ATE }<20 \text { ANOS IDADE } \\
44 \text { PACIENTES }\end{array}$ & $\begin{array}{c}\text { N.DE } \\
\text { PACIENTES }\end{array}$ & $\begin{array}{l}\text { PERC. } \\
\%\end{array}$ & $\begin{array}{c}\text { APOS }>20 \text { ANOS IDADE } \\
340 \text { PACIENTES }\end{array}$ & $\begin{array}{c}\text { N:DE } \\
\text { PACIENTES }\end{array}$ & $\underset{\%}{\text { PERC. }}$ \\
\hline Endocardite & 7 & 45 & Endocardite & 11 & 30 \\
\hline Calcificação & 4 & 29 & Calcificação & 8 & 22 \\
\hline Embolia & 0 & 0 & Embolia & 2 & 6 \\
\hline Insuf. paravalvular & 1 & 6,5 & Insuf, paravalvular & 3 & 9 \\
\hline Insuf. cardiaca & 2 & 13 & Insuf. cardiaca & 6 & 16,5 \\
\hline Arritmias & 1 & 6,5 & Arritmias & 6 & 16.5 \\
\hline TOTAL & 15 & 100 & TOTAL & 36 & 100 \\
\hline
\end{tabular}


VRANDECIC, M. O.: GONTIJO FILHO, B.; SILVA, J. A. P.; FANTINI, F. A.; BARBOSA, J. T.; SĀO JOSÉ, M. C.: PINTO, C. A S.; VIEIRA, G. L.; OLIVEIRA, H. G.; RABELO, R. R.; RABELLO, S. C.; BRICK, A. V.; PEREDO, E.; PEDROSA, A. A.: AZEVEDO SOBRINHO, A. L. O.; BARBOSA, M.; MIOTTO, H. C.; BRAGA, M. A.; SALUM, M. A.; BRAGA, J. F.; MOREIRA, G. H.; MOREIRA. O. H.; OLIVEIRA, C. A.; MACIEL, F. J. - Estudo multicêntrico dos resultados das trocas valvares com o uso da bioprotese Biocor no Estado de Minas Gerais. Rev. Bras. Cir. Cardiovasc., 3(3): 159-168, 1988.

assinalam 6 eventos fulminantes de discrasia sangüínea operatória sem causa aparente, assim como insuficiência renal ( 6 casos) e 5 casos de AVC, na Unidade de Cuidados Intensivos. As complicaçōes tardias dos grupos mitral e aórtico estão sumarizados na Tabela 2 e serão particularizadas a seguir.

\section{Tromboses e Tromboembolismo}

Os eventos tromboembólicos são baixos e, na sua maioria, a recuperação é total. Porém, em 2 pacientes do grupo mitral, observou-se tromboembolismo maciço, com óbito, ambos portadores de fibrilação atrial crônica, cardiomegalia e sem uso de anticoagulantes.

A curva atuarial para o grupo aórtico (Gráfico 1) é de $99,4 \%$ e, para os mitrais, de $98,7 \%$, livre de fenômenos tromboembólicos, sem uso de anticoagulantes.

\section{Endocardite}

A partir de 1986 , todo o tecido valvar, seja nativo ou bioprótese, foi enviado para exame anatômico patológico; este estudo, que será objeto de outro trabalho, demonstrou, para nossa surpresa, presença de endocardite ativa em $30 \%$ dos espécimens encaminhados, embora a suspeita clínica ou cirúrgica fosse de falência

GRÁFICO 1

GRUPO AÓRTICO EMBOLIA

CURVA ATUARIAL

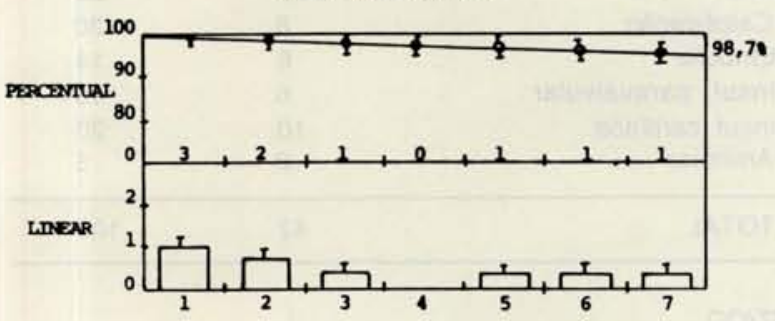

GRUPO MITRAL

EMBOLIA

CURVA ATUARIAL

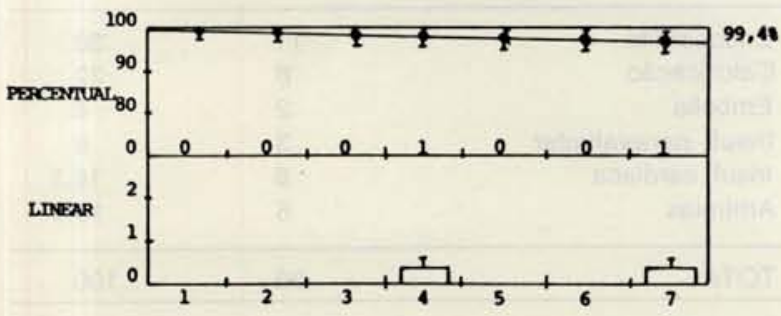

valvar sem evidência macroscópica de infecção. O estudo histológico destes espécimens identificou presença de "nichos" localizados, preferencialmente, nas bordas e, inclusive, dentro de blocos calcificados, evidenciando atividade, sugerida pela presença de infiltrado neutrofilico e depósitos fibrinóides. Estes achados, somados ao quadro clínico apresentado no pós-operatório imediato de alguns pacientes (estado subfebril, taquicardia discreta, certa palidez, anemia relativa, dificil evolução pós-operatória), sugerem maior controle da assepsia em cada procedimento cirúrgico.

A incidência hospitalar de endocardite exuberante é baixa, inclusive nas valvas nativas e biopróteses, cujo exame anatomopatológico sugere focos de endocardite. Os pacientes aórticos estão livres desta complicação em $95,6 \%$ dos casos e os mitrais, em $97,1 \%$ (Gráfico 2).

\section{Falência Valvular Primária}

No grupo mitral, a degeneração espontânea e a fibrocalcificação dos folhetos valvulares suínos foi de $4,74 \%$ (34 em 716).

Nos pacientes menores de 20 anos, a incidência foi de $14,77 \%$ (26 em 176). No grupo aórtico, encontramos um total de 12 calcificaçōes em 385 pacientes $(3,11 \%)$, sendo que $9,09 \%$ ( 4 em 44$)$ ocorreram no grupo

GRÁFICO 2

GRUPO AORTICO

ENDOCARDITE

CURVA ATUARIAL

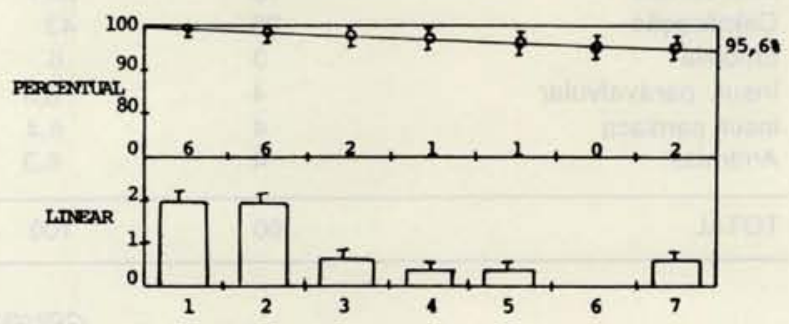

GRUPO MITRAL

ENDOCARDITE

CURVA ATUARIAL

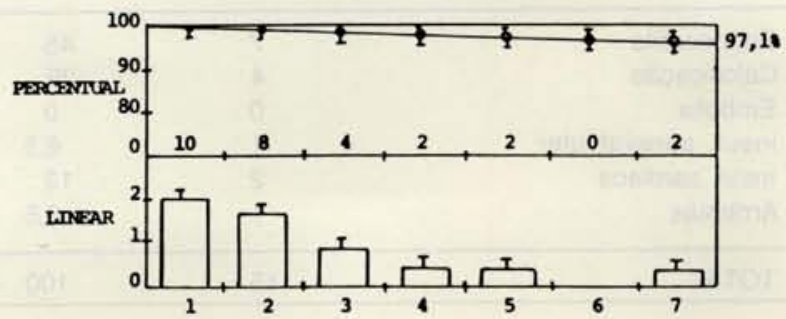


VRANDECIC, M. O.; GONTIJO FILHO, B.; SILVA, J. A. P.; FANTINI, F. A.; BARBOSA, J. T.; SÃO JOSÉ, M. C.; PINTO, C. A. S.; VIEIRA, G. L.; OLIVEIRA, H. G.; RABELO, R. R.; RABELLO, S. C.; BRICK, A. V.; PEREDO, E.; PEDROSA, A. A.; AZEVEDO SOBRINHO, A. L. O.; BARBOSA, M.; MIOTTO, H. C.; BRAGA, M. A.; SALUM, M. A.; BRAGA, J. F.; MOREIRA, G. H.; MOREIRA, O. H.; OLIVEIRA, C. A.; MACIEL, F. J. - Estudo multicêntrico dos resultados das trocas valvares com o uso da bioprótese Biocor no Estado de Minas Gerais. Rev. Bras. Cir. Cardiovasc., 3(3): 159-168, 1988.

de idade inferior a 20 anos. A curva atuarial do grupo aórtico mostra $96,9 \%$ de pacientes livres de falência valvular. No grupo mitral, esta percentagem atinge $95,2 \%$ (Gráfico 3) e, nos pacientes menores de 20 anos, $85,3 \%$ (Gráfico 4).

\section{GRÁFICO 3}

GRUPO AORTICO

LIVRE DE FALEANCIA VALVULAR CURVA ATUARIAL

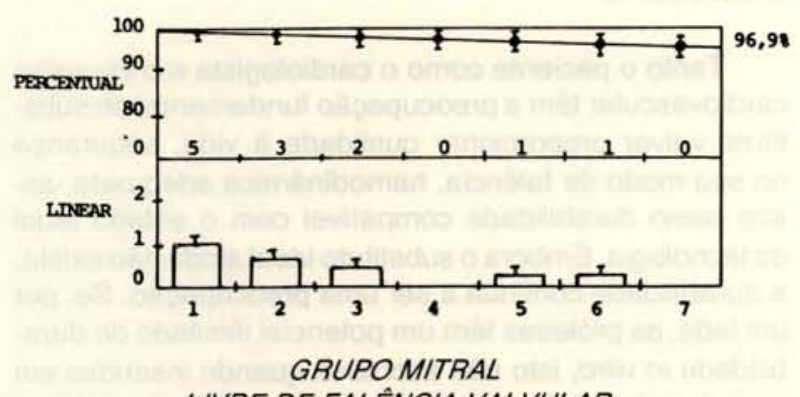

LIVRE DE FALENCIA VALVULAR CURVA ATUARIAL

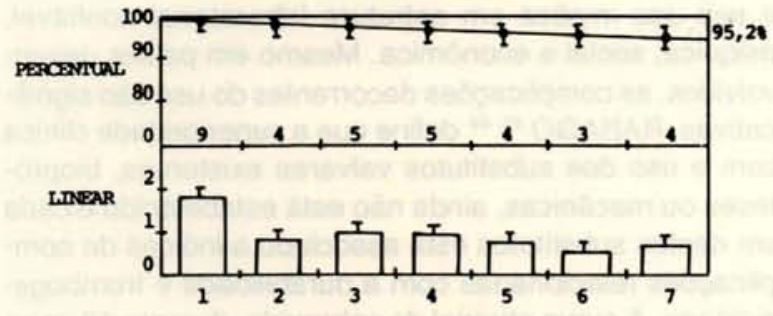

GRÁFICO 4

GRUPO MITRAL

LIVRE DE FALENCIA VALVULAR $<20$ ANOS CURVA ATUARIAL
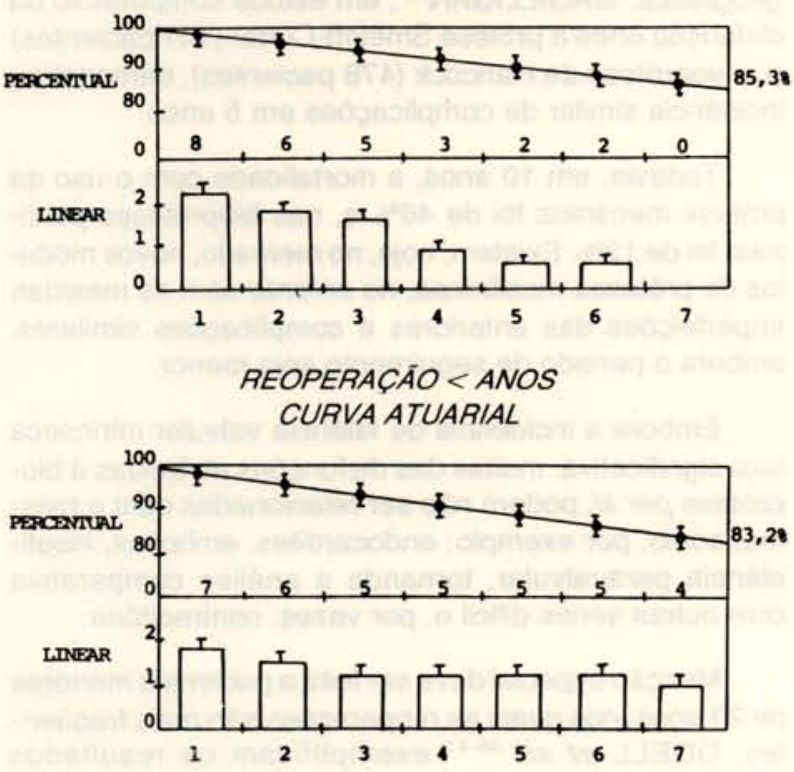

TABELA 3

GRUPO AORTICO

COMPLICAÇŌES DETERMINANDO REOPERAÇĀO

\begin{tabular}{lccc}
\hline & $<$ 20 ANOS & $>20$ ANOS & TOTAL \\
\hline Endocardite & 2 & 4 & 6 \\
Calcificação & 4 & 8 & 12 \\
Insuf. paravalvular & 0 & 2 & 2 \\
\hline TOTAL & 6 & 14 & 20 \\
\hline
\end{tabular}

GRUPO MITRAL

COMPLICAÇÕES DETERMINANDO REOPERACCÃO

\begin{tabular}{lrcc}
\hline & $<20$ ANOS & $>20$ ANOS & TOTAL \\
\hline Endocardite & 9 & 5 & 14 \\
Calcificação & 20 & 5 & 25 \\
Insuf. paravalvular & 2 & 1 & 3 \\
\hline TOTAL & 31 & 11 & 42 \\
\hline
\end{tabular}

TABELA 4

RESULTADOS CIRÚRGICOS EM 62 REOPERACÓES DE ACORDO COM O GRAU FUNCIONAL

\begin{tabular}{lccc}
\hline & $\begin{array}{c}\text { N. PACIENTES } \\
\text { OPERADOS }\end{array}$ & $\begin{array}{c}\text { MORTALIDADE } \\
\text { OBITOS }\end{array}$ & $\%$ \\
\hline Classe II & 12 & 0 & 0 \\
Classe III & 30 & 2 & 8 \\
Classe IV & 20 & 5 & 30 \\
\hline TOTAL & 62 & 7 & 12,6 \\
\hline
\end{tabular}

\section{Reoperação}

Vários pacientes necessitaram de retroca da válvula, 42 no grupo mitral e 20 no grupo aórtico. As complicaçōes determinantes de reoperação, em ambos os grupos, foram endocardite e falência intrínseca da bioprótese (calcificação) (Tabela 3 ). A probabilidade de reoperação aumenta a partir do 5 : ano, observando-se uma média de reoperação de $16,8 \% \pm 8,4 \%(37 / 220)$ nos pacientes menores de 20 anos e de $2,8 \% \pm 9,3 \%$ (26/881) nos pacientes acima de 20 anos. A incidência da mortalidade, na reoperação, é diretamente proporcional ao grau funcional do paciente (Tabela 4), sendo a mortalidade global de $12,6 \%$. A curva atuarial dos pacientes menores de 20 anos livres de reoperação é de $83,2 \%$ (Figura 4 ).

\section{Mortalidade relacionada à bioprótese}

Inclui a mortalidade global das complicações relacionadas ao uso da bioprótese. Sete pacientes faleceram 
VRANDECIC, M. O.; GONTIJO FILHO, B.; SILVA, J. A. P.; FANTINI, F. A.; BARBOSA, J. T.; SÃO JOSÉ, M. C.; PINTO, C. A. S.; VIEIRA, G. L.; OLIVEIRA, H. G.; RABELO, R. R.; RABELLO, S. C.; BRICK, A. V.; PEREDO, E.; PEDROSA, A. A.; AZEVEDO SOBRINHO, A. L. O.; BARBOSA, M.; MIOTTO, H. C.; BRAGA, M. A.; SALUM, M. A.; BRAGA, J. F.; MOREIRA, G. H.; MOREIRA, O. H.; OLIVEIRA, C. A.; MACIEL, F. J. - Estudo multicêntrico dos resultados das trocas valvares com o uso da bioprótese Biocor no Estado de Minas Gerais. Rev. Bras. Cir. Cardiovasc., 3(3): 159-168, 1988.

em decorrência da reoperação, 2 pacientes por tromboembolismo maciço, 20 por endocardite, sem intervenção cirúrgica. A insuficiência cardiaca foi responsável por 5 óbitos e as arritmias, por 2. Ocorreram 5 óbitos por disfunção da prótese, em pacientes não reoperados, por diversos motivos. A curva atuarial dos grupos aórtico e mitral livre desta complicação foi de $97,1 \%$ (Gráfico 5).

GRÁFICO 5

LIVRE DE MORTALIDADE RELACIONADA Aं BIOPROTTESE GRUPO MITRAL E AORTICO CURVA ATUARIAL
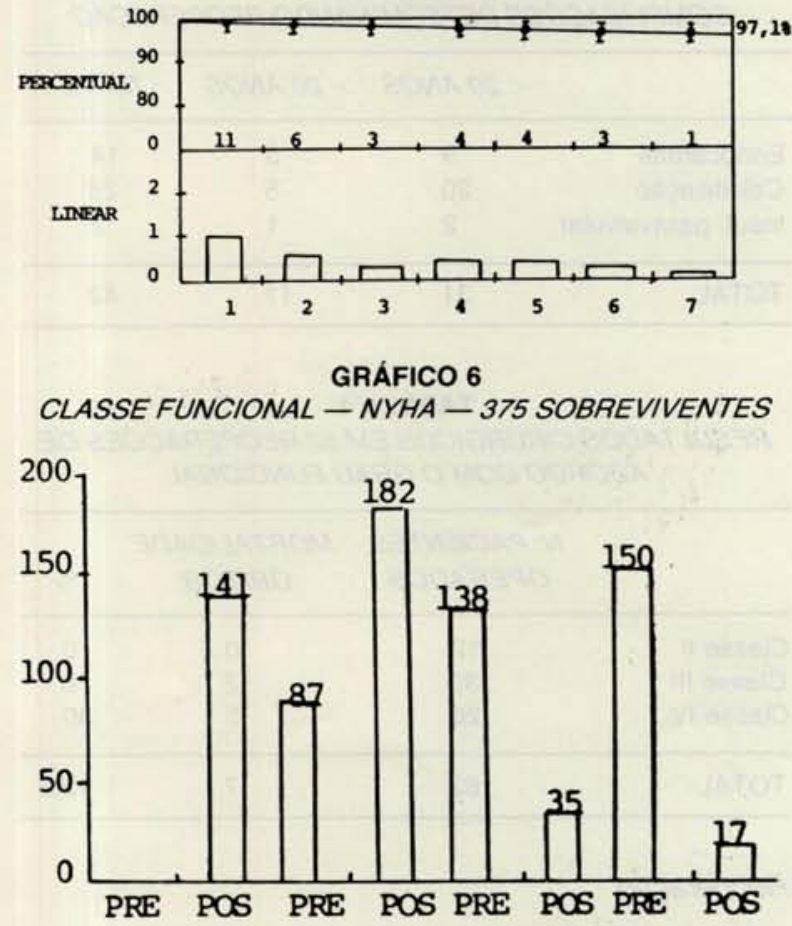

I

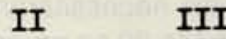

IV

GRUPO MITRAL

CLASSE FUNCIONAL - NYHA - 694 SOBREVIVENTES

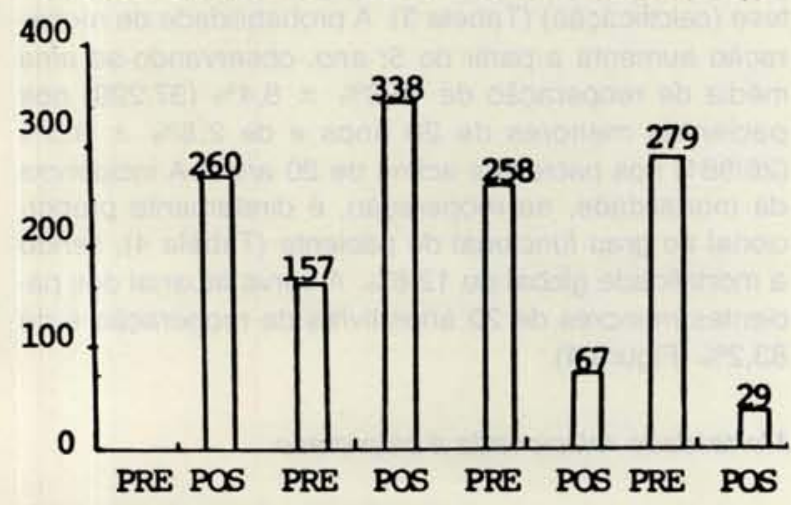

I

II

III

IV

\section{Classificação funcional pós-operatória}

Dos 1069 pacientes (aórticos 375/mitrais 694), 85\% encontram-se em classe funcional I e II, estando em classe funcional III $10 \%$ e $5 \% \mathrm{~cm}$ classe funcional IV (Gráfico 6).

\section{DISCUSSĀO}

Tanto o paciente como o cardiologista e o cirurgiāo cardiovascular têm a preocupaçāo fundamental do substituto valvar proporcionar qualidade à vida, segurança no seu modo de falência, hemodinâmica adequada, assim como durabilidade compatível com o estado atual da tecnologia. Embora o substituto ideal ainda nāo exista, a durabilidade continua a ser uma preocupação. Se, por um lado, as próteses têm um potencial ilimitado de durabilidade in vitro, isto nāo acontece quando inseridas em posição intracardiaca, onde o meio sangüineo exige o uso de anticoagulantes que, de per si, são iatrogênicos e seu uso implica em estrutura laboratorial confiável, psíquica, social e econômica. Mesmo em países desenvolvidos, as complicaçōes decorrentes do uso são significativas. RABAGO ${ }^{43,44}$ define que a superioridade clínica com o uso dos substitutos valvares existentes, biopróteses ou mecânicas, ainda não está estabelecido e cada um destes substitutos está associado a índices de complicaçōes relacionadas com a durabilidade e trombogenicidade. A curva atuarial de sobrevida, durante 13 anos de evolução, mostra $72 \% \pm 2 \%$ para as próteses mecânicas e de $78 \% \pm 2 \%$ para as biopróteses. Sugerindo que a escolha deva ser baseada, principalmente, levando em consideraçāo o aspecto sócio-econômico da área geográfica, MAGILLIGAN ${ }^{27}$, em estudo comparativo da disfunção entre a prótese Smeloff-Cutter (107 pacientes) e a bioprótese de Hancock (478 pacientes), demonstrou incidência similar de complicações em 5 anos.

Todavia, em 10 anos, a mortalidade com o uso da prótese mecânica foi de $46 \%$ e, nas biopróteses porcinas, foi de $13 \%$. Existem, hoje, no mercado, novos modelos de próteses mecânicas, no entanto com as mesmas imperfeiçōes das anteriores e complicaçōes similares, embora o período de seguimento seja menor.

Embora a incidência de falência valvular intrínseca seja significativa, muitas das disfunçōes atribuídas à bioprótese per si, podem não ser relacionadas com a mesma, como, por exemplo: endocardites, embolias, insuficiência paravalvular, tornando a análise comparativa com outras séries difícil e, por vezes, contraditória.

Menção especial deve ser feita a pacientes menores de 20 anos, nos quais as reoperaçōes são mais freqüentes. ODELL et alii ${ }^{38-41}$ exemplificam os resultados 
VRANDECIC, M. O.; GONTIJO FILHO, B.; SILVA, J. A. P.: FANTINI, F. A.; BARBOSA, J. T.; SÃO JOSÉ, M. C.; PINTO, C. A S.; VIEIRA, G. L.; OlIVEIRA, H. G.; RABELO, R. R.; RABELLO, S. C.; BRICK, A. V.; PEREDO, E.; PEDROSA, A. A.; AZEVEDO SOBRINHO, A. L. O.; BARBOSA, M.; MIOTTO, H. C.; BRAGA, M. A.; SALUM, M. A.; BRAGA, J. F.; MOREIRA, G. H.; MOREIRA, O. H.; OLIVEIRA, C. A.; MACIEL, F. J. - Estudo multicêntrico dos resultados das trocas valvares com o uso da bioprótese Biocor no Estado de Minas Gerais. Rev. Bras. Cir. Cardiovasc., 3(3): 159-168, 1988.

obtidos em 768 pacientes até 15 anos, com o uso de próteses mecânicas e biopróteses, demonstrando a sobrevida cumulativa durante 10 anos de $73,2 \% \pm 3,9 \%$ para as próteses mecânicas e de $62,6 \% \pm 5,3 \%$ para as biopróteses. A trombose e o tromboembolismo são raros no quadro das biopróteses, sendo que apenas 41,9 $\pm 5,2 \%$ das próteses mecânicas estão livres desta complicação, durante o período de 10 anos. A calcificação nas biopróteses de porcino, nessa série, é de $37,2 \%$, 14,5 meses após o implante (média). Nas biopróteses de pericárdio bovino, é de $28,7 \%$ em apenas 7,4 meses (média). Esta maior incidência de calcificação das biopróteses de pericárdio bovino ${ }^{17}, 27,37,42,44,46$, sugerida do ponto de vista clínico e laboratorial, seria devida à calcificação ser intrínseca e extrínseca, ao contrário das biopróteses de suíno, onde a calcificação é apenas intrínseca ${ }^{9,10}$. A presença exagerada de depósitos de fibrina no tecido de pericárdio bovino, iniciaria uma fina camada de trombo, dias após o implante, acelerando o processo de calcificação. Em nosso material, a incidência de calcificaçāo nos pacientes com idade abaixo de 20 anos, durante os 7 anos de seguimento ( $m=48$ meses), foi de $13,63 \%(30 / 220)$. Neste grupo de pacientes, a endocardite, em geral, se apresenta nos primeiros 24 meses; portanto, é fundamental a determinação da indicaçâo cirúrgica o mais cedo possivel, como aconselhado por MILLER $^{32}$, MILLE \& OYER ${ }^{33}$ e SPENCER et ali $^{44}$, para se obterem melhores resultados.

Muito se tem discutido sobre a escolha do substituto valvar no grupo etário jovem; contudo, a realidade é a escolha entre o uso de uma bioprótese cuja durabilidade é limitada, ou a prótese de incidência tromboembólica exagerada MITHA ${ }^{35}$.

A incidência de mortalidade relacionada a uso da bioprótese inclui todas as causas relacionadas à falência da bioprótese, inclusive endocardites. Todavia, este dado de vital importância só seria real se pudessem ser feitas necropsias em cada caso. As limitaçōes neste sentido são enormes e, do ponto de vista comparativo, favorecem as próteses mecânicas, pois mais de $50 \%$ dos episódios de tromboembolismo fatal em próteses são somente identificadas em necropsia ${ }^{36}$.

Em nosso material, a sobrevida livre de mortalidade relacionada à bioprótese em posição aórtica e mitral foi de $97,1 \pm 7,2 \%$ (32/1101), ao cabo de 7 anos (Gráfico 5).

Neste grupo etário jovem, se a calcificação pode ser retardada, o uso de bioprótese de porcino proporcionará resultados aceitáveis, como em adultos. Com este objetivo, os Laboratórios Biocor possuem um método específico de seleção de cúspides aórticas suínas em frigoríficos qualificados, processando-se as mesmas apenas minutos após o abate; esta seleção de cúspides desprovidas de banda muscular é de 300 para 1 bioprótese. $\mathrm{O}$ controle da homogenicidade do processamento é feito por lotes e inclui microscopia de luz e eletromicroscopia. A média do processamento é superior a 3 meses, incluindo análises bacteriológicas a cada etapa do mesmo. O rigor do teste mecânico é individual, usando, como parâmetros, medida de gradientes, bem como a funcionabilidade de cada folheto. Testes de durabilidade em lotes são feitos usando o duplicador de pulso acelerado, que permite, em poucas semanas, a reprodução do número de ciclos correspondentes a 5 anos. $\mathrm{O}$ ingrediente básico do processamento tem sido a solução de glutaraldeído e surfactante; todavia, pesquisas em andamento demonstram que a mesma não é insubstituivel e outras soluçōes são capazes de produzir crosslinkage do colágeno e a preservação dos elementos intrínsecos da zona esponjosa proteoglican 48,49 etc, essenciais para prolongar a durabilidade e retardar a calcificação. Neste sentido, os testes de durabilidade, usando o duplicador de pulso com este processo específico, tem aumentado a vida média de $21 / 2$ vezes. $O$ implante destas biopróteses realizado pelo Professor Michael P. Kaye, da Universidade de Minnesota Cardiovascular Research Laboratories, confirma os testes in vitro, obtendo-se o bom funcionamento sem calcificação, após 3 meses.

Aguardamos a confirmação de outros Centros, para poder iniciar o teste clínico deste produto, cuja base histoquímica consiste no uso de soluções capazes de retardar a calcificaçāo. CARPENTIER ${ }^{8}$ e IMAMURA \& KITAMURA ${ }^{23}$ enfatizam que o futuro das biopróteses depende da nossa habilidade em obter meios que diminuam gradientes transvalvulares, aumentem a durabilidade, retardando ao máximo a calcificação, de modo especial nos jovens.

Estudos comparativos de gradientes transvalvulares realizados por BLACK ${ }^{5}$, entre biopróteses Biocor, Hancock, Carpentier-Edwards, lonescu-Shiley e outras, demonstram menores gradientes, especialmente no diâmetro 23 Biocor, devido à técnica de montagem, usando 3 cúspides selecionadas, desprovidas de banda muscular; este estudo in vitro foi confirmado por BARBOSA ${ }^{4}$. Melhor durabilidade e diminuição da calcificação são prioridades que vêm sendo estudadas e já em estágio final.

As complicações tromboembólicas, embora presentes, são complicaçōes de menor magnitude, assim como as insuficiências paravalvulares. A incidência de endocardite, semelhante nas próteses, teve um relativo aumento no nosso estudo, em função do tipo de paciente, cujas defesas naturais, em geral, estāo diminuídas.

A degeneração das biopróteses de suíno é real; todavia é rara a sua contribuição para eventos catastróficos, proporcionando quase sempre a oportunidade para as retrocas em bases eletivas, ou, pelo menos, urgentes. 
VRANDECIC, M. O.; GONTIJO FILHO, B.; SILVA, J. A. P.; FANTINI, F. A.; BARBOSA, J. T.; SÃO JOSÉ, M. C.; PINTO, C. A. S.; VIEIRA, G. L.; OLIVEIRA, H. G.; RABELO, R. R.; RABELLO, S. C.; BRICK, A. V.; PEREDO, E.; PEDROSA, A. A.; AZEVEDO SOBRINHO, A. L. O.; BARBOSA, M.; MIOTTO, H. C.; BRAGA, M. A.; SALUM, M. A.; BRAGA, J. F.; MOREIRA, G. H.; MOREIRA, O. H.; OLIVEIRA, C. A.; MACIEL, F. J. - Estudo multicêntrico dos resultados das trocas valvares com o uso da bioprótese Biocor no Estado de Minas Gerais. Rev. Bras. Cir. Cardiovasc., 3(3): 159-168, 1988.

\section{CONCLUSÕES}

A qualidade de vida e a expectativa de sobrevida, com o uso das biopróteses, é superior ao uso de próteses. em nosso meio, a impossibilidade do uso adequado de anticoagulantes é uma limitação importante.

A incidência de fenômenos tromboembólicos, com o uso das biopróteses, é aceitavelmente baixa e as complicações catastróficas são raras. A falência estrutural (desgaste do próprio tecido), no adulto, é inferior a $20 \%$ durante 10 anos; todavia, as reoperaçōes têm baixa mortalidade, quando realizadas em paciente em classe funcional II. A qualidade de vida é melhor, o modo progressivo de falência e a maior expectativa de vida justificam o uso das biopróteses.

O potencial, a curto prazo, é de substancial durabilidade e o retardamento do início da calcificação, usando novas técnicas de preservação, fixaçāo em repouso (sem uso de pressāo), soluçōes outras que o glutaraldeido, com o objetivo de obter o processamento de todos os componentes da estrutura da cúspide, deverão reduzir os processos degenerativos, prolongando a durabilidade.

A mortalidade, associada às reoperaçōes, pode ser diminuída, já que é estritamente em função da preservação da função miocárdica. O controle sistemático destes pacientes e a identificação das complicações não só são possiveis, mas é responsabilidade da familia, do paciente e do médico.

Embora o substituto ideal continue sendo pesquisado, os resultados deste trabalho e da literatura mundial demonstram que o uso da Bioprótese Biocor tem proporcionado qualidade de vida, durabilidade esperada e baixa mortalidade, neste estudo multicêntrico, justificando seu uso clínico.

RBCCV 44205-62

VRANDECIC, M. O.; GONTIJO FILHO, B.; SILVA, J. A. P.; FANTINI, F. A.; BARBOSA, J. T.; SĀO JOSÉ, M. C.; PINTO, C. A. S.; VIEIRA, G. L.; OLIVEIRA, H. G.; RABELO, R. R.; RABELLO, S. C.; BRICK, A. V.; PEREDO, E.; PEDROSA, A. A.; AZEVEDO SOBRINHO, A. L. O.; BARBOSA, M.; MIOTTO H. C.; BRAGA, M. A.; SALUM, M. A.; BRAGA, J. F.; MOREIRA, G. H.; MOREIRA, O. H.; OLIVEIRA, C. A.; MACIEL, F. J. - Multicentric study of the results with implanted bioprosthesis Biocor in the state of Minas Gerais. Rev. Bras. Cir. Cardiovasc., 3(3): 159-168, 1988.

ABSTRACT: From March 1981 to March 1988, 2324 bioprostheses were implanted in 2016 patients in 5 centers in the State of Minas Gerais. This study includes only the analysis of patients undergoing isolated aortic $(n=603)$ or mitral $(n=1110)$ valve replacement. The hospital mortality in this group $(n=1713)$ was 104 patients $(6.1 \%)$. From the remaining 1609 patients, that were discharged from the hospital, we were able to obtain a follow-up in 1101 or $64.3 \%$. This analysis related to a period ranging from 1 to 84 months, mean $=48$ and a cumulative follow-up in the aortic group $(n=385)$ equal to 1230 patients/year; the mitrals $(n=716$ ) equal to 3018 patients/year. There were 102 late complications in 716 patients (14.24) and 51 in the aortic group (13.2). In relation to the age or the patients, 220 were under 20 years of age (mitrals $=176$ /aortics $=44$ ) and in this subgroup the intrinsic valve failure was $43 \%$ of the mitral patients and $29 \%$ of the aortic group. The prosthetic endocardite was more frequent in the aortic group $(45 \%)$ in compararison with the mitrals $(29.7 \%)$. There were 62 reoperations in 1101 patients, with hospital mortality of $12.6 \%$. The reoperations were more frequent in the mitral group, in patients below 20 years of age. Survival, considering only deaths related to the bioprosthesis, was $97.1 \%(32 / 1101)$. In the aortic group, $96.9 \%$ of patients were free of valve disfunction at the end of 7 years; among the mitrals, $95.2 \%$. The major incidence of valve failure was encountered in the mitral patients, below 20 years of age; $85.3 \%$ were free of this complication at 7 years of follow-up. At the end of this study, the majority of the alive patients was functional classes I and II of the NYHA. Although the incidence of reoperation is significant, these findings are acceptable, specially because bioprosthesis offers a safer alternative than mechanical prosthesis. The present results suggest the continuation of research to obtain the ideal bioprosthesis, although current data is comparable to results abtained with similar bioprosthesis in the world literature.

DESCRIPTORS: heart valves, biologic; heart valves prostheses, surgery; heart valves, surgery. 
VRANDECIC, M. O.; GONTIJO FILHO, B.; SILVA, J. A. P.; FANTINI, F. A.; BARBOSA, J. T.; SÃO JOSÉ, M. C.; PINTO, C. A. S.; VIEIRA, G. L.; OLIVEIRA, H. G.; RABELO, R. R.; RABELLO, S. C.; BRICK, A. V.; PEREDO, E.; PEDROSA, A. A.; AZEVEDO SOBRINHO, A. L. O.; BARBOSA, M.; MIOTTO, H. C.; BRAGA, M. A.; SALUM, M. A.; BRAGA, J. F.; MOREIRA, G. H.; MOREIRA, O. H.; OLIVEIRA, C. A.; MACIEL, F. J. - Estudo multicêntrico dos resultados das trocas valvares com o uso da bioprótese Biocor no Estado de Minas Gerais. Rev. Bras. Cir. Cardiovasc., 3(3): 159-168, 1988.

\section{REFERÊNCIAS BIBLIOGRÁFICAS}

1 ANTUNES, M. J. - Bioprosthetic valve replacement in children: long-term follow-up of 135 isolated mitral valve implantation. Eur. Heart J., 5(8): 913-915, 1984.

2 ANTUNES, M. J. - Thromboembolic complication and anticoagulants: compliant versus noncompliant patient populations. In: RABACO, G. \& COOLEY, D. A. Heart valve replacement \& future trends in cardiac surgery. Mount Kisco, NY, Futura Publishing Co. Inc., 1987. p. 307-317.

3 ANTUNES, M. J. \& SANTOS, L. P. - Performance of glutaraldehyde preserved porcine bioprosthesis as a mitral valve substitute, in a young population group. Ann. Thorac. Surg., 37(5): 387-392, 1984.

4 BARBOSA, M. R. - Avaliação hemodinâmica da prótese heteróloga Biocor. Arq. Bras. Cardiol., 42(2). 123-128, 1984.

5 BLACK, M. M. - Accelerated fatigue testing of biological tissue heart valves. Proc. ESAO, 7(2): 315-319, 1980.

6 BOLOOKI, H.; MALLON, S.; KAISER, G. A. - Failure of Hancock xenograft valve: importance of valve position (4-to 9-year follow-up). Ann. Thorac. Surg., 36(3): 246-252, 1983

7 BORTOLOTTI, U.; MILANO, A.; MAZZUCCO, A. - Results of reoperation for primary tissue failure of porcine bioprostheses. J. Thorac. Cardiovasc. Surg., 90(4): 564-600, 1982.

8 CARPENTER, A. - Current status and the future of tissue valves. In: DURAN, C. \& ANGELL, W. W. Recent progress in mitral valve disease. England, Butterworth \& Co. Ltd., 1984. p. 234-243.

9 COHN, L. H.: ALFRED, E. N.; DISESA, V. J. - Early and late risk of aortic valve replacement: a 12 year concomitant comparison of the porcine bioprosthetic and tilting disc prosthetic aortic valve. J. Thorac. Cardiovasc. Surg., 88(5, Parte 1): 695-705, 1984.

$10 \mathrm{COHN}$, L. H.;-MUDGE, G. H.; PRATTER, F. - Five to eight year folow-up of patients undergoing porcine heart valve replacement. N. Engl. J. Med., 304(2):258-262, 1981.

11 CONTI, V. R.; NISHIMURA, A.; COUGHLIN, T. R.; FARRELL, R. W. - Indications for replacement of the Beall 103 and 104 disc valves. Ann. Thorac. Surg., 42(3): 315-320, 1986.

12 CZER, L. S. \& MATLOFF, J. M. - Experience with the St. Jude valve. In: RABAGO, G. \& COOLEY, D. A. Heart valve replacement \& future trends in cardiac surgery. Mount Kisco, NY, Futura Publishing Co. Inc. 1987. p. 153-166.
13 EMERY, R. W.; METTLER, E.; NICOLOFF, D. M. - A new cardiac prosthesis: the St. Jude Medical cardiac valve. In vivo results. Circulation, 60(Supl. 1): 48 , 1979.

14 FENNERTY, M. B.; PICK, R. A.; CLARK, M. A. - Outlet strut fracture of a Björk-Shiley prosthesis in the mitral position. Am. J. Cardiol., 54(10): 923-931, 1984.

15 FERNANDEZ, J.; SAMUEL, A.; YANG, S. S. - Late thrombosis of the aortic Björk-Shiley prosthesis: its clinical recognition and management. Chest, 70(1): 12-16, 1976.

16 FUSTER, V.; PUMPHREY, C. W.; McGOON. M. D. Systemic thromboembolism in mitral and aortic StarrEdwards prostheses: 10-19-year follow-up. Circulation, 66(Supl. 1): |-157-|-161, 1982

17 GALlO, I; NISTAL, F.; REVUELTA, J. M. - Incidence or primary tissue valve failure with lonescu-Shiley pericardial valve. J. Thorac. Cardiovasc. Surg., 90(2): 278-280, 1985.

18 GALLO, I.; RUIZ, B.; NISTAL, F.; DURAN, C. M. G. Degeneration in porcine bioprosthetic cardiac valves: incidence of primary tissue failure among 938 bioprostheses at risk. Am. J. Cardiol., 53(11): 1061-1066. 1984.

19 GALLUCCI, V.; VALFRE, C.; MAZZUCCO, A. - Heart valve replacement with the Hancock bioprosthesis: a 5-11 year follow-up. In: COHN, L. H. \& GALLUCCI, V. Cardiac bioprostheses: Proceedings of the Second International Symposyum. New York, Yorke Medical Books, 1982. p. 9-24.

20 GEHA, A. S.; HAMMOND, G. L.; LAKS, H. - Factors affecting performance and thromboembolism after porcine xenograft cardiac valve replacement. J. Thorac. Cardiovasc. Surg., 83(4): 377-384, 1982.

21 GRAY, R.; CHAUX, A.; MATLOFF, J. - Early postoperative hemodynamic comparison of St. Jude cardiac prostheses and porcine xenografts, at rest and with stress. Circulation, 59, 60(Suppl. 2): 222, 1979. (Resumo).

HIRSCH, J.; DEYKIN, D.; POLLER, L. - "Therapeutic range" for oral anticoagulant therapy. Chest, 89(Supl.): 11S, 1986.

23 IMAMURA, E. \& KITAMURA, M. - A trial of fixation modification for more physiological performance: preliminary hemodynamic evaluation open-fixation of bioprosthesis. In: GABAY, S. \& CABROL, C. Calcification and degeneration of heart valve bioprosthesis. Paris, A. Ameur, 1988. p. 247-262.

24 JAMIESON, W. R. E.; GEREIN, A. N.; RICCI, D. R. Carpentier-Edwards supra-annular porcine bioprosthesis: a new generation tissue valve (clinical and hemodynamic assessment). In: BODNAR, E. \& YACOUB, M. 
VRANDECIC, M. O.; GONTIJO FILHO, B.; SILVA, J. A. P.; FANTINI, F. A.; BARBOSA, J. T.; SĀO JOSÉ, M. C.; PINTO, C. A. S.; VIEIRA, G. L.; OLIVEIRA, H. G.; RABELO, R. R.; RABELLO, S. C.; BRICK, A. V.; PEREDO, E.; PEDROSA, A. A.; AZEVEDO SOBRINHO, A. L. O.; BARBOSA, M.; MIOTTO, H. C.; BRAGA, M. A.; SALUM, M. A.; BRAGA, J. F.; MOREIRA, G. H.; MOREIRA, O. H.; OLIVEIRA, C. A.; MACIEL, F. J. - Estudo multicêntrico dos resultados das trocas valvares com o uso da bioprótese Biocor no Estado de Minas Gerais. Rev. Bras. Cir. Cardiovasc., 3(3): 159-168, 1988.

Biologic and bioprosthetic valves: Proceedings of the Third International Symposium. New York, Yorke Medical Books, 1986. p. 141.

JAMIESON, W. R. E.; MUNRO, A. I.; MacNAB, J.; PATTERSON, M. W. H. - Porcine bioprostheses in patients 20 years and younger: is there a role for this prosthesis? In: BODNAR, E. \& YACOUB, M. Biologic and bioprosthetic valves: Proceedings of the Third International Symposium. New York, Yorke Medical Books, 1986. p. 290.

KINSLEY, R. H.; COLSEN, P. R.; ANTUNES, M. J. Medtronic-Hall valve replacement in a third-world population group. J. Thorac. Cardiovasc. Surg., 31(2): $69-74,1983$

27 MAGILLIGAN, Jr., D. J. - Advantages and disadvantages of tissue valves. In: STAREK, P. J. K. Heart valve replacement and reconstruction. London, Year Book Medical Publishers Inc., 1987. p. 237-245.

28 MAGILLIGAN Jr., D. J.; HEINZERLING, R. H.; SMITH D. - Fate of a second porcine bioprosthetic valve. J. Thorac. Cardiovasc. Surg., 85(3): 362-370, 1983.

MAGILLIGAN Jr., D. J. \& TILLEY, B. - The porcine bioprosthetic valve: twelve years later. J. Thorac. Cardiovasc. Surg., 89(4): 499-507, 1985.

30 MARTINELL, J.; FRAILE, J.; ARTIZ, V. - Long-term comparative analysis of the Björk-Shiley and Hancock valves implanted in 1975 . J. Thorac. Cardiovasc. Surg., 90(4): 741-749, 1985

31 MILANO, A.; BORTOLOTTI, U.; TALENTI, E. - Calcific degeneration as the main cause of porcine bioprosthetic valve failure. Am. J. Cardiol., 53(8): 1066-1070, 1984.

MILLER, D. C. - Performance characteristics of the StarrEdwards model 1260 aortic valve prosthesis beyound ten years: editorial rebuttal. J. Thorac. Cardiovasc. Surg., 88(2): 203-207, 1984

MILLER, D. C. \& OYER, P. E. - Ten-year clinical experience in 1651 patients with one type of tissue valve. In: STAREK, P. J. K. Heart valve replacement and reconstruction. London, Yeark Book Medical Publishers Inc., 1987. p. 175-189.

34 MILLER, D. C.; OYER, P. E.; MITCHELL, R. S. - Performance characteristics of the Starr-Edwards model 1260 aortic valve prosthesis beyond ten years. J. Thorac. Cardiovasc. Surg., 88(2): 193-207, 1984.

MITHA, A. S. - Clinical experience with the Lillenhei-Kaster cardiac prosthesis. J. Thorac. Cardiovasc. Surg., 72(3): 401-407, 1976.

MURPHY, D. A.; LEVINE, F. H.; BUCKLEY, M. J. - Mechanical valves: a comparative analysis of the Starr-Ed- wards and Björk-Shiley prostheses. J. Thorac. Cardiovasc. Surg., 86(5): 746-752, 1983.

37 ODELL, J. A. - Experience with tissue and mechanical valves in the pediatric age group. In: RABAGO, G, \& COOLEY, D. A. Heart valve replacement \& future trends in cardiac surgery. Mount Kisco, NY. Futura Publishing Co. Inc., 1987. p. 185-208.

38 OYER, P. E.; MILLER, D. C.; STINSON, E. B. - Clinical durability of the Hancock porcine bioprosthetic valve. J. Thorac. Cardiovasc. Surg., 80(6): 824-833, 1980

39 OYER, P. E.; STINSON, E. B.; MILLER, D. C. - Clinical analysis of the Hancock porcine bioprosthesis. In: COHN, L. H. \& GALLUCCI, V. Cardiac bioprosthesis: Proceedings of the Second International Symposium. New York, Yorke Medical Books, 1982. p. 539-551.

40 OYER, P. E.; STINSON, E. B.; MILLER, D. C. - Thromboembolic risk and durability of the Hancock bioprosthetic cardiac valve. Eur. Heart J., 5(Supl. D): 81-85, 1984.

41 PASS, H. J.; CRAWFORD, F. A.; HOHN, A. R. - Cardiac valve replacement in children without anticoagulation. J. Thorac. Cardiovasc. Surg., 87(6): 832-835, 1984

42 RABAGO, G. - Comparison of mechanical and biological prosthesis. In: RABAGO, G. \& COOLEY, D. A. Heart valve replacement \& future trends in cardiac surgery. Mount Kisco, NY, Futura Publishing Co. Inc., 1988. p. $245-256$

43 RABAGO, G. - A worldwide overview of valve usage In: RABAGO, G. \& COOLEY, D. A. Heart valve replacement \& future trends in cardiac surgery. Mount Kisco, NY, Futura Publishing Co. Inc., 1987. p. 3-9.

44 SPENCER, F. C.; GROSSI, E. A.; CULLIFORD, A. T. Experiences with 1643 porcine prosthetic valves in 1942 patients. Ann. Surg., 203: 691-700, 1986

45 STEIN, P.; COLLINS, J. J.;KANTROWITZ, A. - Antithrombotic therapy in mechanical and biological heart valves and saphenous vein bypass grafts. Chests., 89(Supl.): 46S, 1986

46 THUBRIKOR, M. J.; DECK, J. D.; AOUD, J. - Role of mechanical stress in calcification of aortic bioprosthetic valves. J. Thorac. Cardiovasc. Surg., 86(1):115-125, 1983.

47 VRANDECIC, M. O. P. - Clinical experience with a new generation of porcine bioprosthesis. In: BODNAR, E. \& YACOUB, M. Biologic bioprosthetic valves: Proceedings of the Third International Symposium. New York, Yorke Medical Books, 1986. p. 559-665.

48 VRANDECIC, M. O. P. - New bioprosthesis design. J. Cardiovasc. Surg., 28(Supl. 5): 18, 1987. 